\section{The conjunction fallacy? A reply to Bar-Hillel}

\section{GEORGE WOLFORD \\ Dartmouth College, Hanover, New Hampshire}

In her commentary on our article, Bar-Hillel (1991) argues that our normative interpretation of the Linda problem is inaccurate and that the representativeness heuristic can account for all of the relevant data. I argue that the normative interpretation of the Linda problem remains slippery and that representativeness fails to account for much of the data. Furthermore, substantial evidence supports our suggestion that subjects use different underlying models in estimating likelihoods in different conjunction problems.

Wolford, Taylor, and Beck (1990) showed that it is possible to construct conceptually similar problems that require different normative models for correct solution. We referred to those models as the "known" and the "unknown" models. With the unknown model, rating a conjunction as more likely than one of its constituents is a fallacy; with the known model, a conjunction can be more likely than one of its constituents. By using examples in which the choice of the appropriate model is unambiguous (to experts), we showed that subjects are sensitive to the distinction. We also argued that the choice of the appropriate model is ambiguous in some cases and that some of the problems traditionally used to study the conjunction fallacy (e.g., the Linda problem) fall into that category. Therefore, we reasoned that some of the conjunction fallacies with those traditional problems may have resulted from the subjects' assuming that the known model applied.

\section{Choice of Normative Model}

In her commentary, Bar-Hillel agrees that there are two distinct problem types but prefers to recast the distinction in terms of conditional probabilities. She disagrees that the Linda problem is ambiguous with respect to the appropriate normative model. Finally, she contends that the representativeness heuristic is sufficient to account for all of the data relevant to the Linda problem. We shall discuss each of those agreements and disagreements in turn.

As mentioned above, Bar-Hillel (1991) agrees that there are two distinct problem types. However, she does not accept the unknown versus known models as ways of characterizing that distinction. Instead, she argues that the distinction is best framed in terms of different conditional probabilities (e.g., $P(B \& F \mid L)$ vs. $P(L \mid B \& F)$ ). We accept her characterization. In fact, in our original arti-

Correspondence should be addressed to the author at the Department of Psychology, Dartmouth College, Hanover, NH 03755. cle, we acknowledged that characterization as a distinction between conjunctive events and conjunctive evidence (p. 49), but Bar-Hillel stated the distinction more clearly than we did. (Henceforth, I will refer to $P(B \& F \mid L)$ as conjunctive events and $P(L \mid B \& F)$ as conjunctive evidence.)

Although we agree that her description of the two normative cases is both clear and apt, we were trying to capture something further with our known versus unknown models, namely, the differences in circumstances appropriate to the use of one or the other of the two normative models. Experimenters working on the Linda problem rarely use the explicit formalisms of probability theory in describing the problem. ${ }^{1}$ Instead, they use everyday language to communicate better with the subjects, but this may create ambiguity. Additional difficulties are that much of the research done on the Linda problem is carried out on large groups (e.g., introductory classes) and that the published descriptions often contain limited information about the exact instructions, the number of problems used with a single group, the context in which the problem or problems were administered, and so forth.

To illustrate the difficulty in choosing the correct model, I will describe various possible readings of the Linda problem. Assume that three alternatives are listed: bank teller, feminist, and the conjunction. If the instructions asked subjects to rate either the likelihood of bank teller and/or feminist given Linda or the likelihood of Linda given bank teller and/or feminist, then the appropriate normative model is clear. But the instructions are rarely so explicit. From the literature, the most common instruction is something like "rate the likelihood of the following alternatives." What if the subject believes that there is a correct answer (i.e., that one and only one of the alternatives is "true")? This poses a problem for the conjunctive-events interpretation. Under the extension rule (conjunctive-events model), it is quite possible to have more than one true answer. In fact, under the extension rule, if the conjunction is true, then both of the other alternatives must be true. With the conjunctive-evidence interpretation, it is possible for any of the alternatives to be the only true answer.

It seems to us that the typical instructions afford more than one possible interpretation. We stated in the original article that the conjunctive-events interpretation seemed most appropriate but that we also believe the conjunctive-evidence interpretation is possible. Since the problem and outcomes are rarely framed in formal terms, what cues are available to the subject for deciding among possible models? One of our first thoughts was that if the outcome has not yet happened, then only the conjunctiveevents model is appropriate. The Borg and RGRRR problems (Tversky \& Kahneman, 1983, pp. 302-304) make it clear that the outcomes lie in the future. Unfortunately, "future" is not an adequate characterization. If 
the outcome has occurred, but nobody knows what it is, then the conjunctive-events model is still unambiguously correct. We chose to name this condition as "unknown," for lack of a better term. Unfortunately, "known" is a less adequate characterization for the converse of what we were trying to capture with the unknown model. Contrary to Bar-Hillel's claim, however, it is not necessary to mention another person in the Linda problem for the conjunctive-evidence model to apply. If the subject is led to believe that one and only one of the alternatives is Linda, then the conjunctive-evidence model is a reasonable choice. The subject might choose to assume that the other alternatives are associated with other individuals, but that is not necessary for the model to apply.

\section{Accounting for the Relevant Data}

Putting aside the question of whether there is one and only one correct interpretation of the Linda problem, is there any evidence that some subjects are influenced by a conjunctive-evidence interpretation of the problem? There is. We provided several lines of converging evidence that showed that subjects are influenced by the distinction. First, we showed that when the context is elaborated to make the choice of underlying model unambiguous, subjects' judgments are affected (and quite significantly so). The most interesting comparisons are between what we refer to as "Linda-Control" and "Linda-Unknown." The former is one of the standard versions of the Linda problem; the latter provides context that demands the use of the conjunctive-events (unknown) interpretation. According to Bar-Hillel, these two should be essentially identical, since she argues that the normative model is unambiguous in the control problem, as well as in the unknown version. In fact, subjects made the "conjunction fallacy" far more often in the control version: $82 \%$ versus $58 \%$ with introductory psychology students and $90 \%$ versus $53 \%$ with medical students. The differences were even more marked with students from the two populations who had had some statistical training ( $82 \%$ vs. $43 \%$ and $89 \%$ vs. $41 \%$ ). These differences were all highly significant, with chisquare values ranging from a low of 6.40 to a high of 10.95 and $p$ values ranging from .011 to .001 .

Second, we pointed out that there were differences in the rates of conjunction fallacies in the work of Tversky and Kahneman (1983). Some problems were less ambiguous than others (to us) in the choice of the correct interpretation. For instance, we argued that the Linda problem was somewhat ambiguous and the Borg problem was unambiguous. In the most comparable versions of the Linda and Borg problems, $85 \%$ of 142 students made the fallacy with the Linda problem and $72 \%$ of 93 students with the Borg problem. That difference yields a chi-square of 6.09 and a $p$ value of .013 . Other problems in their paper followed the general relationship of less ambiguity/fewer conjunction fallacies.

Third (and related to the preceding point), we had 12 relatively sophisticated judges (psychology faculty and ad- vanced graduate students) rate various problems from Tversky and Kahneman (1983) as to the ambiguity of the choice of normative model. We briefly mentioned some of the ratings in the original article. We began by explaining the two normative models to the judges and had them rate several problems on a 7-point scale in which 1 indicated a problem involving conjunctive evidence (known model) and 7 indicated a problem involving conjunctive events (unknown model). Their ratings were consistent with our judgments that some problems, such as Borg (average rating $=6.6$ ) and RGRRR (6.0), clearly implied conjunctive events and some, such as Linda (2.4), Bill (2.6), and dyspnea (3.8), were ambiguous. Those differences in rating were highly significant, yielding an $F(4,44)$ $=9.63, p$ near 0 . As mentioned above, the first two problems (Borg and RGRRR) yielded significantly fewer conjunction errors than did the last three.

Fourth, there is a fascinating difference in Tversky and Kahneman (1983) in subjects' ability to recognize the appropriate argument when confronted with it. We mentioned that contrast in our original article, but I will summarize it again here. Bar-Hillel (1991) claims that experts recognize the error of their ways in the Linda problem when they are confronted with appropriate arguments. Somewhat different results are presented in Tversky and Kahneman (1983). They presented 58 undergraduates with two statements describing two ways of thinking about the Linda problem: one was the conjunctive-events argument, and the other was the representativeness argument. Sixtyfive percent of the students failed to choose the conjunctive-events argument-Tversky and Kahneman (1983) interpreted this as further evidence for the strength of the conjunction fallacy. Since we believe that the problem is ambiguous, we are not surprised by the outcome. Later in the article, they presented two similar arguments concerning the RGRRR problem to 88 undergraduates. This time only $24 \%$ of the students failed to recognize the correct argument. We suggest that the RGRRR problem is unambiguous with respect to the correct normative model and are not surprised by the huge shift from the Linda problem. The difference between the two results yields a chi-square of 25.19 , with a vanishingly small $p$ value.

We do not see how an account based solely on representativeness can account for the above results. It is equally true that some of the data cannot be accounted for by ambiguity in the choice of models. Even when the conjunctive-events model is clear, many subjects make a conjunction fallacy. In most areas of psychological research, we recognize that complex behavior may result from a number of processes. In the judgment literature, for some reason, there appears to be a desire to have one process per behavior. I am confident that judgments in problems such as the Linda problem will turn out to be the result of several factors. One of those factors will surely be something akin to representativeness. I am convinced that some of the variance will be shown to result from differences in the choice of the underlying model. 


\section{Prediction}

Bar-Hillel (1991) writes, "When subjects judge the probability of a conjunction ... to lie between the probabilities of the component events, we have nice evidence [that they are using] the logic of similarity, which predicts just such an order" (p. 000). I would like to quibble with the suggestion that similarity or representativeness has "predictive" value. Prediction involves the advance specification of an outcome. The people who investigate the role of representativeness in judgment have added qualifications that seem to make prediction difficult. Tversky and Kahneman (1983, p. 296) discussed a couple of examples involving female body weight and Hollywood actresses in which the large majority of students recognized that the less representative event was more probable. That is, students do not always judge likelihood based on representativeness. Furthermore, Tversky and Kahneman (1983) write later in the same article, "Such observations imply that the judged probability (or representativeness) of a conjunction cannot be computed as a function (e.g., product, sum, minimum, weighted average) of the scale values of its constituents" (p. 305). Bar-Hillel (1982) argues that in complex cases, it might be difficult to determine similarity (or representativeness) and that one might want to use likelihood judgments to aid in determining similarity. I do not use these examples to imply that representativeness is not an important variable in uncertainty judgments, but I do believe that the construct is not sufficiently well specified to afford prediction.

\section{Conclusions}

Representativeness clearly plays a role in people's judgments in uncertain situations. I do not believe, however, that it is sufficient to account for all of the data. I further believe that many subjects' judgments have been influenced by the choice of a normative model other than the one intended by the experimenter. Further research is necessary to elucidate all of the factors that influence judgments in related problems.

\section{REFERENCES}

BAR-HilleL, M. (1982). Studies of representativeness. In D. Kahneman, P. Slovic, \& A. Tversky (Eds.), Judgment under uncenainty: Heuristics and biases. New York: Cambridge University Press.

BAR-Hillel. M. (1991). Commentary on Wolford, Taylor, and Beck: The conjunction fallacy? Memory \& Cognition, 19, 412-414.

Tversky, A., KahNeman, D. (1983). Extensional versus intuitive reasoning: The conjunction fallacy in probability judgment. Psychological Review, 90, 293-315.

Wolford, G., TAYLOR, H., BECK, J. R. (1990). The conjunction fallacy? Memory \& Cognition, 18, 47-53.

\section{NOTE}

1. Interestingly, Tversky and Kahneman (1983) point out that subjects rarely make the conjunction fallacy when the problem is stated in abstract terms. It is possible that the abstract terms make it clear that a conjunctive-events interpretation is needed.

(Manuscript accepted for publication November 2, 1990.) 